\section{"3-D": An interactive computer-graphics program for plotting scores on three coordinate axes}

\author{
WAYNE P. ASPEY \\ Marine Biomedical Institute \\ University of Texas Medical Branch, Galveston, Texas 77550 \\ RUSSELL W. ZEARS \\ Division of Communications and \\ Educational Support Services \\ University of Texas Medical Branch, Galveston, Texas 77550
}

and

\author{
JAMES E. BLANKENSHIP \\ Marine Biomedical Institute and \\ Department of Physiology \& Biophysics \\ University of Texas Medical Branch, Galveston, Texas 77550
}

Program " $3-\mathrm{D}$ " is an interactive computer-graphics package designed to assist the researcher in achieving a functional, mathematically accurate, and aesthetically pleasing graphical representation of data that require plotting in three dimensions. The program plots data from a variable number of groups on three coordinate axes such that the data appear displayed in three dimensions. Furthermore, the program allows the $\mathrm{X}$ and $\mathrm{Y}$ coordinate axes to be shifted to the left or right and up or down in order to achieve the best graphical representation (i.e., group separation) of the three-dimensional space.

Description. Program "3-D" consists of a main program that drives nine subroutines and interfaces with the user. Subroutine 1 (COR) coordinates the mapping of $\mathrm{X}, \mathrm{Y}$, and $\mathrm{Z}$ axes onto a two-dimensional display plane. Two-point perspective vanishing points are used to provide an aesthetically pleasing illusion of threedimensionality. Vanishing points are empirically determined according to the data and/or the user's needs.

Examples of left/right and up/down shifts of the $X$ and $Y$ axes are illustrated in Figure 1. Subroutine 2 (STARTP) initializes plotting by setting the size of the graph and determining its center as a reference for the pen coordinates. For our needs, the window size was empirically determined as $25 \times 25 \mathrm{~cm}$. Subroutine 3 (ENDP) ends the plot package and makes the program listing more readable. Subroutine 4 (LABELP) is used by Subroutine 9 (AXESP) to position and plot the labels along the three axes. Subroutine 5 (WLINE) plots a line specified in three-dimensional space, and is used by both Subroutines 7 (BALOON) and 9 (AXESP). Subroutine 6 (SYMBP) selects and plots a symbol for each input group at the appropriate three-dimensional point. Subroutine 7 (BALOON) uses Subroutines 5 (WLINE) and 6 (SYMBP) to plot the data points and the corresponding lines that extend to the $X-Y$ plane. Subroutine 8 (RECOR) recoordinates, or rescales, the data points to the proper three-dimensional coordinate values for two-dimensional representation. Finally, Subroutine 9 (AXESP) functions as graph paper by drawing the entire three-dimensional grid floor and three axes, and inserting the labels.

Input. The computer requests the following usersupplied information: (1) vanishing points for the $X$, $Y$, and $Z$ coordinate axes in the format $(-X, Z)$ and $(Y, Z) ;(2)$ number of groups to be plotted; (3) number of items in Group 1; (4) Group 1 data entered as a series of three coordinate values for each subject; (5) number of items in Group 2; (6) Group 2 data; and continues until data for all groups have been entered. Labels up to 12 characters each can be added to Subroutine 9 (AXESP).

Output. Figure 1 (A) illustrates sample output using data from Aspey and Blankenship (1976, 1977). The viewing angle is determined by the vanishing points, and Figure 1 (B-D) illustrates various "grid floor" tilts and $X$ and $Y$ axes shifts to the left and right. For a display window of $25 \times 25 \mathrm{~cm}$, the vanishing points listed in Figure 1 (C and D) represent the best maximum limit shifts to the left and right, respectively, for our system. Users can empirically determine those vanishing points best suited for their data or needs.

Restrictions. Program " $3-D$ " is restricted in the size of graphical output as a function of the available printing equipment. With our system, only eight symbols are available for representing the different input groups. Additionally, the array of possible vanishing points becomes more restricted as the overall size of the graph increases.

Computer and Language. Program " $3-D$ " is written in FORTRAN IV and was developed with a DEC PDP-1 1/45 computer at the Marine Biomedical Institute. Hard-copy printout is provided by a Gould 5000 plotter.

Availability. Copies of this paper, a descriptive source listing of the program, and documented output from an illustrative example are available at no charge on request to Dr. Wayne P. Aspey, Division of Comparative Neurobiology \& Behavior, Marine Biomedical Institute, University of Texas Medical Branch, 200 University Boulevard, Galveston, Texas 77550. 


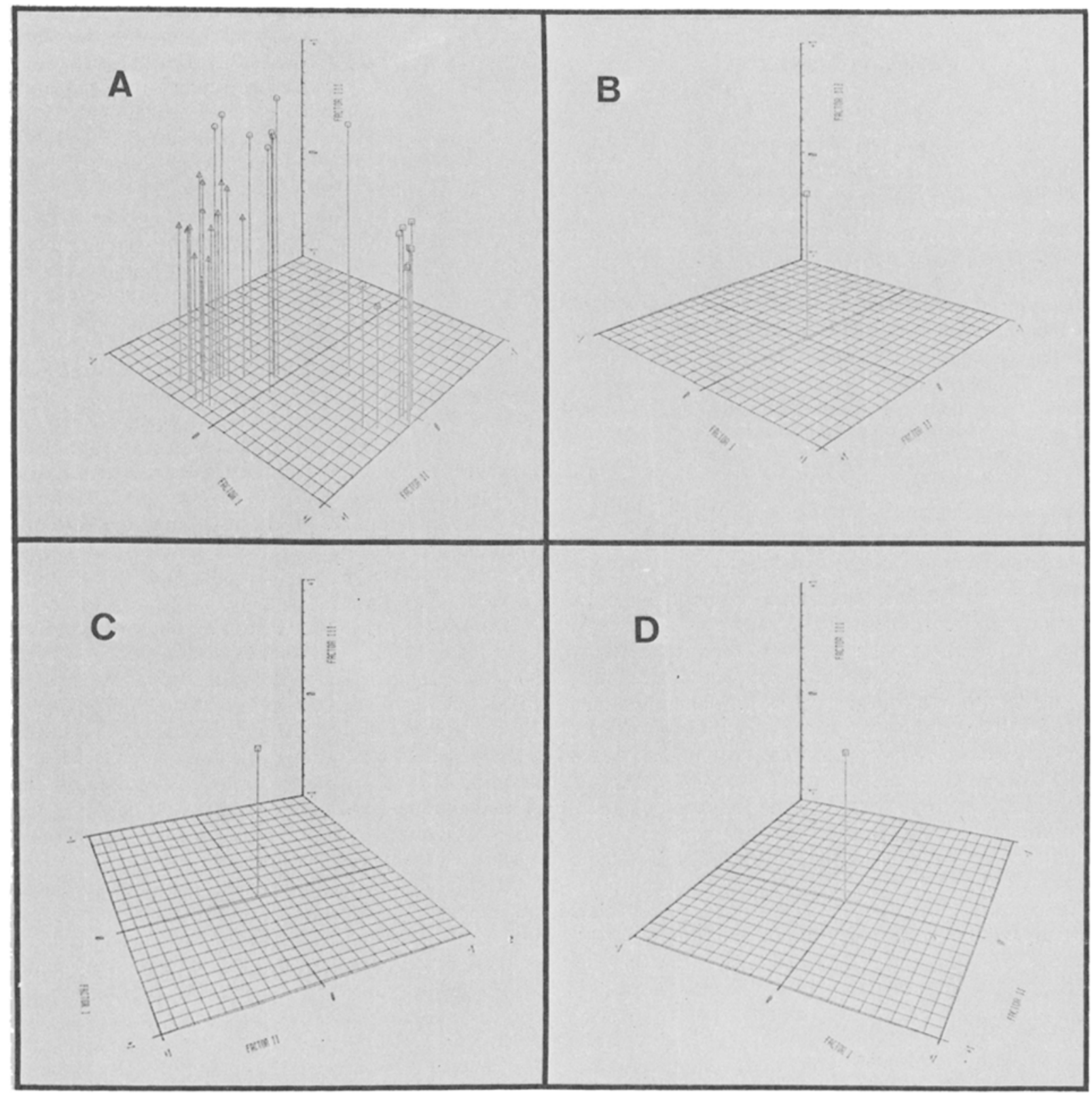

Figure 1. Computer-generated three-dimensional mapping of data projected onto two-dimensions as plotted by program " 3 -D". The $\mathrm{X}$ and $\mathrm{Y}$ coordinate axes are plotted in the horizontal plane, while the $\mathrm{Z}$ coordinate axis projects vertically. The origin $(0,0,0)$ falls in the center of the three-dimensional space, and is plotted in B, C, and D; labels along the edges of the coordinate axes facilitate plotting. (A) Data from Aspey and Blankenship $(1976,1977)$ replotted by computer using two-point perspective vanishing points of $(-14.8505,6.0)$ and $(11.7757,6.0)$ to approximate the human artist rendition of the data. (B) The "grid floor" can be raised or lowered by altering the vanishing points of the $Z$ coordinate axis. Vanishing points $(-14.8505,4.3)$ and $(11.7757,4.3)$ raise the floor while keeping the left-right shift of the $X$ and $Y$ axes the same as in $A$. (C) The grid floor can be shifted to the left or right so as to best accommodate the data. Vanishing points $(-6.0,5.0)$ and $(26.0,5.0)$ were empirically selected to provide the best maximum limit $X$ and $Y$ axes shift to the left; floor tilt was intermediate between $A$ and $B$. (D) Vanishing points $(-26.0,5.0)$ and $(6.0,5.0)$ provide the best maximum limit $X$ and $Y$ axes shift to the right with the same floor tilt as in $C$.

\section{REFERENCES}

Aspey. W. P. d Blankenship, J. E. Aplysia behavioral biology: I. A multivariate analysis of burrowing in $A$. brasiliana. Behavioral Biology, 1976, 17, 279-299.

Aspey, W. P.. \& Blankenship, J. E. Spiders and snails and statistical tales: Applications of multivariate analyses to diverse ethological problems. In B. A. Hazlett (Ed.). Quantitative methods in the study of animal behavior. New York: Academic Press, 1977. Pp. 75-120.

(Accepted for publication May 20, 1977.) 